\title{
Erratum to "Overcoming the bottleneck of platelet lysate supply in large-scale clinical expansion of adipose-derived stem cells: A comparison of fresh versus three types of platelet lysates from outdated buffy coat-derived platelet concentrates" [Cytotherapy 2017;19:222-234]
}

\author{
PETER V. GLOVINSKI, MIKKEL HERLY, ANDERS B. MATHIASEN, \\ JESPER D. SVALGAARD, REHANNAH BORUP, MAJ-LIS M. TALMAN, JENS J. ELBERG, \\ STIG-FREDERIKT KØLLE, KRZYSZTOF T. DRZEWIECKI \& ANNE FISCHER-NIELSEN
}

In the above-mentioned published work, as a response to one of the reviewers request for the amount of population doublings (PDs), we calculated the PDs for the results section in addition to the population doubling times (PDT). However the text was never changed accordingly and states PDT instead of PDs in the section of results.

Proliferation Assay (page 5): "Although growth in all three types of O-PLs re-sulted in growth rates of P1 ASCs that were not significantly different from growth in F-PL, there was a trend toward a lower PDT for O-PL-InterSol, $\mathrm{P}=0.06$ (Figure 2). The PDTs of the ASCs were. ......."

The sentence should instead read: "Although growth in all three types of O-PLs resulted in growth rates of P1 ASCs that were not significantly different from growth in F-PL, there was a trend toward fewer population doublings (PDs) for O-PL-InterSol, $\mathrm{P}=0.06$. The PDs of the ASCs were. ......."

In the first section of the Discussion (page 8) the same mistake is also found: "For all three types of PLs generated from outdated PCs, we found PDTs that were comparable to that of fresh PL, although there was a trend towards a lower PDT for the O-PLInterSol (Figure 2).

The sentence should instead read: "For all three types of PLs generated from outdated PCs, we found PDTs that were comparable to that of fresh PL, although there was a trend towards a fewer PDs for the O-PL-InterSol (Figure 2). 